\section{MicroRNAs in mouse models of lymphoid malignancies}

\author{
Nicola Zanesi, ${ }^{1}$ Yuri Pekarsky, ${ }^{1}$ Francesco \\ Trapasso, ' George Calin, ${ }^{2}$ Carlo M. Croce ${ }^{1}$ \\ 'Department of Molecular Virology, \\ Immunology and Medical Genetics, Ohio \\ State University, Columbus, $\mathrm{OH}$; \\ ${ }^{2}$ Department of Experimental \\ Therapeutics and Center for RNA \\ Interference and Non-Coding RNAs, \\ University of Texas M.D. Anderson \\ Cancer Center, Houston, TX, USA
}

\section{Abstract}

The discovery of microRNAs (miRNAs) has revealed a new layer of gene expression regulation that affects many normal and pathologic biological systems. Among the malignancies affected by the dysregulation of miRNAs, there are cancers of lymphoid origin in which miRNAs are thought to have tumor suppressive or tumor promoting activities, depending on the nature of their specific targets. In the last four to five years, the experimental field that provided the deepest insights into the in vivo biology of miRNAs is that of mouse modeling, in which transgenic and knockout animals mimic over-expression or down-regulation, respectively, of specific miRNAs involved in human leukemia/ymphoma. This review discusses recent advances in our understanding of lymphoid malignancies based on the natural and engineered mouse models of three different miRNAs: miR-15a/16-1 cluster, miR155, and miR-17-92 cluster.

\section{Introduction}

MicroRNAs (miRNAs) are a class of endogenous non-coding RNAs, 19-25 nucleotides in size, which regulate gene expression at either the transcriptional or the post-transcriptional level. ${ }^{1}$ MiRNAs bind to messenger RNAs (mRNAs) primarily at their 3 ' untranslated regions (UTRs) via partial complementarity and, consequently, mRNA translation and/or stability are impaired, ultimately resulting in a reduction in protein expression levels. Recent studies have shown that miRNAs are important in development and in processes such as cellular differentiation, growth, cell death, ${ }^{2}$ and DNA methylation. ${ }^{3}$ The first finding linking miRNAs and cancer was that chronic lymphocytic leukemias (CLL) often exhibit deletions or down-regulation of two miRNAs: miR-15a and miR-16-1, at 13q14. ${ }^{4}$
Many annotated human miRNAs are located at fragile sites and genomic regions non-randomly altered in specific cancers, ${ }^{5}$ suggesting that miRNAs could function as tumor suppressors and oncogenes. ${ }^{6}$

Leukemia is a heterogeneous malignancy characterized by the abnormal proliferation of blood precursor cells of myeloid or lymphoid origin and the number one cancer killer of children younger than 14 years of age. To date, a number of miRNAs involved in leukemia have been reported and miRNA expression signatures associated with cytogenetics and the clinical outcome of CLL, acute myeloid leukemia (AML), Hodgkin's lymphoma, ${ }^{2}$ and multiple myeloma ${ }^{7}$ have been identified.

There are two main reasons to model cancer in mice. The first is to determine the causal relationships of specific genetic alterations found in cancer. The second is to engineer defined tumor models for use in studies involving imaging technologies and testing novel therapies. ${ }^{8}$ This review will focus on how some of these approaches have been applied recently to three of the most successful stories in understanding the role of miRNAs in cancers of lymphoid origin: the miR-15/16 cluster as tumor suppressor in CLL, the miR-155 in the creation of the first oncogenic miRNA transgenic mouse, and the miR-17-92 cluster in the generation of the correspondent transgenic mouse (Table 1).

\section{MicroRNA-15a/16-1 cluster}

CLL, the most common leukemia among adults in Western countries, is characterized by progressive accumulation of mostly non-dividing $\mathrm{CD}^{+} \mathrm{B}$ lymphocytes in the blood, bone marrow, and lymphoid tissues. A cluster of miRNAs (namely, miR-15a/16-1) is located in the $13 q 14.3$ region, which undergoes hemizygous or homozygous deletion in more than $50 \%$ of CLLs. ${ }^{9}$ Deletion or down-regulation of expression of this gene cluster in $68 \%$ of CLLs versus normal $\mathrm{CD}^{+}$lymphocytes was reported in $2002{ }^{4}$ A subsequent report showed that the tumor suppressor effect of miR-15a/16-1 is mediated, at least in part, through targeting of $\mathrm{Bcl} 2$, an anti-apoptotic protein. ${ }^{10}$ Interestingly, because $\mathrm{Bc} 22$ is over-expressed in the majority of CLL malignant lymphocytes, but other genetic alterations have not been observed to explain this aberrancy (except in $<5 \%$ of cases), control of Bcl2 expression by the miR-15a/16-1 cluster can be considered as one of the main pathogenic mechanisms of indolent CLL. ${ }^{9}$

Mouse models are valuable tools in the study of human CLL. The New Zealand Black (NZB) strain is a naturally occurring model of lateonset CLL characterized by B cell hyperproliferation and autoimmunity early in life, fol-
Correspondence: Nicola Zanesi, $460 \mathrm{~W} 12^{\text {th }}$ Avenue - Biomedical Research Tower, Columbus, OH 43210, USA.

E-mail: nicola.zanesi@osumc.edu

Key words: mir-15a/16-1, miR-155, miR-17-92, transgenic, knockout.

Contributions: NZ and CMC designed the structure of the review; NZ, YP, and FT wrote the manuscript; GC and CMC edited it; all the authors approved the final version for publication.

Acknowledgements: the authors thank Dr. Kay Huebner for critically reviewing the manuscript and Aaron Burch for technical assistance. NZ is a Kimmel Scholar. This paper has been funded by NIH.

Conflict of interest: the authors report no conflicts of interest.

Received for publication: 27 February 2010.

Revision received: 10 May 2010.

Accepted for publication: 12 May 2010.

This work is licensed under a Creative Commons Attribution 3.0 License (by-nc 3.0).

(C) Copyright N. Zanesi et al., 2010

Licensee PAGEPress, Italy

Journal of Nucleic Acids Investigation 2010; 1:e8 doi:10.4081/jnai.2010.e8

lowed by progression to CLL. All the other models of CLL are genetically engineered strains induced by the expression of exogenous genes. ${ }^{11,12}$ A subpopulation of B cells called B-1 cells are found mainly in the peritoneal and pleural body cavities and form an innate natural antibody defense. Aged NZB mice exhibit clonal expansion of B-1 cells that resemble human CLL. ${ }^{13}$ To determine loci linked to the development of CLL in NZB mice, a genomewide linkage scan of the NZB loci associated with lymphoma/leukemia was conducted in F1 backcrosses of NZB and the DBA/2 control strain. ${ }^{11}$ Of 202 mice phenotyped for presence or absence of B cell lymphoproliferative disease (LPD), surface marker expression, DNA content, and microsatellite polymorphisms, 74 had disease. The $\mathrm{CD}^{+}, \mathrm{IgM}^{+}, \mathrm{B}^{2} 20^{\text {dull }}$, hyperdiploid LPD was linked to three loci on chromosomes 14, 18, and 19, which are distinct from previously identified auto-immunity associated loci. The locus linked to LPD on the NZB chromosome 14 has synteny with human $13 q 14$, which is not unexpected as up to $50 \%$ of cases of CLL have a loss of human 13q14.3. ${ }^{14-16}$

The mouse chromosome 14 region and the human 13q14 region of synteny contain the same two miRNAs, miR-15a and miR-16-1, located in the intronic region of the DLEU2 gene. Because the region of synteny with mouse genomic marker D14mit160 is the 
human $13 q 14$ region associated with CLL and containing miR-15a/16-1, DNA sequencing of this region in NZB mice was performed. The sequence of DNA from multiple NZB tissues showed a point mutation in the 3 '-flanking region of the precursor miRNA, miR-16-1, similar to the $\mathrm{C} \rightarrow \mathrm{T}$ point mutation found in the miR-16-1 3'-flanking sequence in two CLL patients, of which one was from a family with multiple CLL and breast cancers. ${ }^{17}$ This mutation was not present in other strains of mice, including the nearest relative of NZB, the NZW strain. Analysis of the levels of mature miR-16 in tissues isolated from NZB mice revealed decreased expression levels of this miRNA in lymphoid tissues. Delivery of exogenous miR16 to a NZB malignant CLL cell line resulted in cell cycle alterations, including a decrease in cells in S phase and a G1 arrest. ${ }^{18}$ Thus, until recently, linkage of the miR-15a/16-1 complex and the development of B-LPD in this spontaneous mouse model constituted the main evidence that the altered expression of these miRNAs is an important molecular lesion in CLL, confirming the relevance of the correspondent data described in humans.

Eventually, in January 2010, Klein and colleagues published the further genetic validation that deletion of miR-15a/16-1 leads to CLL in mice. ${ }^{19}$ In order to identify the genetic elements targeted by the putative 13q14 tumor suppressor and to determine their contribution to CLL pathogenesis, they generated transgenic mice that carried conditional alleles that either mimicked the deletion of the minimal deleted region (MDR), previously described in human $\mathrm{CLL}^{14,20}$ and comprising the entire DLEU2 gene, or that specifically deleted the miR-15a/16-1 cluster without affecting the expression of DLEU2. At a young age (2-4 months) homozygous Mdr-/- and miR-15a/161-/- mice showed normal percentages of $B$ and $\mathrm{T}$ cell subpopulations and normal development of lymphoid organs. However, at 12 months of age, Mdr-/- mice showed higher percentages of CD5 ${ }^{+}$B220 B cells among mononuclear cells in the peritoneal cavity ( $50 \%$ versus $15 \%$ in controls). Similar observations were made in miR15a/16-1-/- mice. Thus, deletions of the MDR and miR-15a/16-1 are associated with expansion of $\mathrm{CD}^{+} \mathrm{B} 220$ cells in the peritoneal cavity. In a fraction of mice, clonal $\mathrm{CD}^{+} \mathrm{B}$ cell populations were accompanied by significant infiltration of lymphoid organs with histopathological features resembling human CLL. These mice displayed an enlargement of the splenic white pulp owing to the expansion or accumulation of small lymphocytes with a pattern similar to CLL. Discrete aggregates of small lymphocytes were also observed in the bone marrow. Overall, $27 \%$ of Mdr-/- and 21\% of miR15a/16-1-/- mice developed CLL. Altogether $42 \%$ of Mdr-/- and $26 \%$ of miR-15a/16-1-/- mice between 15 and 18 months of age developed

Table 1. Mouse models of lymphoid cancers owing to miRNA alterations.

\begin{tabular}{lllll} 
MiRNA & Method & Alteration & Phenotype & Refs. \\
miR-16-1 & Natural strain (NZB) & $\begin{array}{l}\text { C } \rightarrow \text { T point mutation } \\
\text { in the 3'-flanking region } \\
\text { of the precursor }\end{array}$ & Late-onset CLL & 18,21 \\
miR-15a/16-1 & Knockout & $\begin{array}{l}\text { MDR deletion, miR } \\
\text { cluster deletion }\end{array}$ & $\begin{array}{l}\text { Indolent CLL and its } \\
\text { associated syndromes }\end{array}$ & 19 \\
\hline miR-155 & Transgenesis & $\begin{array}{l}\text { Over-expression in B } \\
\text { cells }\end{array}$ & ALL-like disease & 29,30 \\
miR-155 & Cell transfer & $\begin{array}{l}\text { Over-expression } \\
\text { in bone marrow cells }\end{array}$ & $\begin{array}{l}\text { Myeloproliferative } \\
\text { disease }\end{array}$ & 23,26 \\
\hline miR-17-92 & Transgenesis & $\begin{array}{l}\text { Over-expression in } \\
\text { B and T cells }\end{array}$ & $\begin{array}{l}\text { Lymphoproliferative } \\
\text { disease }\end{array}$ & \multirow{2}{*}{} \\
\hline
\end{tabular}

NZB, New Zealand Black; CLL, chronic lymphocytic leukemia; MDR, minimal deleted region; ALL, acute lymphoblastic leukemia/ymphoma.

some type of clonal B cell lymphoproliferation. Mdr-/- mice also showed a trend toward developing B cell tumors (24\%), suggesting that monoallelic deletion of the MDR can cause disease. This trend is not evident in miR-15a/161-deleted mice, probably because of the lower penetrance of the phenotype. Mdr-/- mice died earlier than their wild type littermates, suggesting that the homozygous mice eventually succumb to their tumors at an advanced age. Conversely, the survival of $\mathrm{miR}-15 \mathrm{a} / 16-1-/-$ mice was not significantly different from wild type littermates, suggesting that these mice show a milder disease course than their Mdr-/counterparts. In conclusion, both Mdr-/- and miR-15a/16-1-/- mice developed lymphoproliferations with an indolent disease course reminiscent of human CLL, with Mdr-/- mice displaying a more aggressive disease phenotype. The significantly more aggressive phenotype displayed by Mdr-/- mice suggests that additional genetic elements within the MDR locus contribute to the tumor suppressive function. One candidate for such a role is DLEU2 itself. Besides providing the primary transcript for the production of miR-15a/16-1, it produces a spliced cytoplasmic RNA that may have important regulatory functions not yet detected. ${ }^{19}$

To understand the mechanism by which the deletions lead to clonal B lymphoproliferations, Klein et al. ${ }^{19}$ investigated whether miR-15a/16-1 deletions could affect proliferation of mouse $B$ cells. BrdU incorporation assays, which measure active DNA-synthesis, demonstrated that miR-15a/16-1-/- B cells begin to synthesize DNA earlier than wild type B cells. Mitogenstimulated B cells purified from miR-15a/16-1/- or Mdr-/- and wild type mice were analyzed for levels of phosphorylated retinoblastoma ( $p$ $\mathrm{Rb})$ protein, an indicator of cell cycle entry. P$\mathrm{Rb}$ could be detected at earlier time points in both miR-15a/16-1-/- and Mdr-/- compared with wild type B cells. Consistent with this observation, expression of the cell cycle inhibitory protein cyclin-dependent kinase inhibitor p27Kip1 appeared to follow similar kinetics. To further unravel the individual contributions of
DLEU2 versus the MiR-15a/16-1 cluster to the lymphoproliferative phenotype, the authors generated an inducible in vitro system in which the DLEU2 transcript and the miR$15 \mathrm{a} / 16-1$ cluster were separately re-expressed in the human I83E95 cell line that is derived from a 13q14-/- CLL. The results showed that miR-15a/16-1 expressing cells, but not those expressing DLEU2, were impaired in proliferation. Consistent with this observation, miR15a/16-1 expressing cells had a higher fraction of cells in the G0/G1 phase compared with both DLEU2 and the control, as documented by BrdU incorporation assays. Thus, expression of the miR-15a/16-1 cluster seems to control cellular proliferation, possibly by inhibiting the G0/G1 phase transition. Taken together, the results of the knockout and re-expression experiments suggest that the miR-15a/16-1 cluster exhibits negative control of proliferation both in human and mouse B cells. On the contrary, the DLEU2 transcript per se did not affect proliferation of $13 q 14$ homozygous deleted cells. Since their findings suggested that miR-15a/16-1 may affect the G0/G1-S phase transition in B cells, the authors sought to identify candidate G0/G1-S phase-related genes among inferred miR-15a/16-1 targets predicted by different computational target prediction algorithms. Seven proteins (cyclins Ccnd2, Ccnd3, and Ccne1, and cyclin-dependent kinases Cdk4, Cdk6, Chk1, and Mcm5) known to be critically involved in the regulation of the G0/G1-S phase transition, were down-regulated in I83E95 13q14-/- cells upon miR-15a/16-1 expression. Analogously, the same gene products showed higher expression levels in anti-IgM-stimulated miR-15a/16-1deficient mouse B cells. Although it remains to be determined whether the proliferation-associated genes identified represent direct or indirect miR-15a/16-1 targets, the concurrent results obtained in two independent genetically defined miR-15a/16-1 knockout systems strongly indicate that these miRNAs regulate the expression of multiple genes involved in the G0/G1-S phase transition in both human 
and mouse B cells.

In another study, Salerno et al. ${ }^{21}$ sought to define a role for miR-16 in the malignant B-1 cell growth and persistence seen in the NZB model of CLL, by examining the effects of exogenous miR-16 on an NZB-derived malignant B-1 cell line that mimics the late stages of CLL. ${ }^{22}$ First, because the NZB spleen has decreased levels of both miR-15a and miR-16 as compared with non-NZB strain spleens, the authors analyzed sorted subpopulations containing non-malignant and malignant $\mathrm{B}$ cells to determine whether NZB malignant B-1 cells had a selective decrease in miR-16 expression. As anticipated, both NZB B-1 cells and conventional B-2 cells had less than half the amount of miR-16 expression relative to the B-1 and B2 populations from age-matched non-NZB strain spleens, indicating that all B cells from NZB mice are affected by the point mutation in the miR-15a/16-1 locus. ${ }^{21}$

In the NZB spleen, most B-2 cells are in G1, whereas the malignant B-1 cells are cycling. ${ }^{18}$ To determine the effects of miR-16 on growth and cell cycle transit of the malignant B-1 cells, NZB B-1 and non-NZB B cell lines were transfected with miR-15a or miR-16 mimic or a negative control mimic (because miR-16 shares a seed sequence and many predicted targets with miR-15a, cells were also transfected with a miR-15a mimic to test if both miRNAs would have the same effect on the cells). Both NZB and non-NZB B cell lines exhibited an increase in the G1 fraction following transfection with miR-15a and miR-16, yet G1 accumulation and $S$ phase decrease in the NZB B cell line were significantly higher than in the non-NZB B cell line. The NZB cell line was also examined for any selective decrease in miR-16 specific to a cell cycle phase. Analysis of basal levels of mature miR-16 expression revealed that the NZB B cell line had lower levels of miR-16 expression than did the non-NZB B cell line at all stages of the cell cycle, with G1 phase cells having significantly higher levels of miR-16. ${ }^{21}$

Because TargetScan, a miRNA target prediction program, listed cyclin D1, a cell cycle regulator, as a likely target for miR-15a and miR16 in humans and mice, to determine if cyclin D1 is regulated by miR-15a or miR-16, NZB and non-NZB cell lines were transfected with either miRNA mimic or the negative control mimic, and cyclin D1 mRNA and protein levels were evaluated at 24 hours post-transfection. PCR revealed no change in cyclin D1 mRNA levels upon miR-16 addition but flow cytometric analysis detected a substantial decrease in the mean fluorescence intensity of cyclin D1 protein in the NZB B cell line transfected with miR-16 compared with expression in the nonNZB B cell line, thus confirming the post-transcriptional regulation of cyclin D1 by miR-16. ${ }^{21}$

\section{MicroRNA-155}

MiR-155 is one of the most prominent and well-studied immune system miRNAs to date. This molecule is clearly involved in protective immunity when properly regulated, yet contributes to malignant conditions upon dysregulation. ${ }^{23}$ Enhanced expression of miR-155 occurs constitutively in a subset of cancer cells of lymphoid ${ }^{24,25}$ and myeloid ${ }^{26,27}$ origin with the highest level of expression in diffuse large B cell lymphomas. ${ }^{28}$

The first animal model specifically built to assess the impact of miR-155 deregulation in a lymphoid malignancy is the E $\mu$-miR155 transgenic mouse model. ${ }^{29}$ These transgenic mice were generated through expression of $\mathrm{mmu}-$ miR-155 (murine miR-155) under the control of a VH promoter-Ig heavy chain $\mathrm{E} \mu$ enhancer, which becomes active at the pro-B cell stage of B cell development. The animals exhibited splenomegaly as early as three weeks of age and showed leukopenia, with the white blood cell count between three and six months of 4to 7-fold lower than in control age-matched mice. Histopathology of the spleens at three weeks of age featured a consistent atypical lymphoid population invading the red pulp and expanding it. At six months, mice presented a greatly increased malignant lymphoid population with marked atypia and blastic appearance, proliferating in the vascular channels of the red pulp and gradually replacing the white pulp. The overall architecture of the spleens was distorted by lymphoid proliferation while a similar lymphoid population was present in the bone marrow as well. Flow cytometry analysis showed an increase of $\mathrm{B} 220^{\text {low }} / \mathrm{CD} 10^{\text {low }}$ IgM-/CD5-/TCR-/CD43- lymphoid cells in both spleen and bone marrow in the transgenic mice. This phenotype resembles the phenotype of proliferating lymphocytes observed in human acute lymphoblastic leukemia or lymphoblastic lymphoma. ${ }^{29}$ This study was the first in which an miRNA transgenic mouse was produced and the first to demonstrate that cancer can be generated by the deregulation of a single miRNA. Subsequently, a marked myeloproliferative disease was also described, by Baltimore's laboratory, in other mice following adoptive transfer of bone marrow cells overexpressing miR-155. ${ }^{26}$

More recently, Costinean et al. reported detailed characterization of the miR-155 transgenic mouse immunophenotype and described a possible mechanism for the development of these leukemias. ${ }^{30}$ Initially, all transgenic animals show an increase of the $\mathrm{B}^{2} 20^{+} \mathrm{IgM}^{-}$population; later on, these cells tend to lose some of the B220 surface antigen expression and become $\mathrm{B} 220^{\text {low; }}$; thus, the studied leukemias exhibit a mixed immunophenotype characterized by $\mathrm{B}^{2} 20^{\text {low }} \operatorname{IgM}^{-}$and $\mathrm{B} 220^{\text {low }} \operatorname{IgM}^{+}$(most likely owing to some degree of differentiation of some of the malignant clones of the pre-B cells of origin). Moreover, all transgenic mice exhibit an increase of the myeloid line and a reduced $\mathrm{T}$ cell population. To understand the role of miR-155 in B cell differentiation and leukemogenesis, the investigators focused on two proteins known to be both key molecules in $\mathrm{B}$ cell maturation/activation and predicted targets of miR-155, SHIP, and C/EBP $\beta$. Src homology 2 domain-containing inositol-5phosphatase (SHIP) is a negative regulator of cell signaling in the immune system. This phosphatase is also thought to be involved in B cell maturation because it shows differential expression in the pro-B compared to the pre-B stage. ${ }^{31}$ Irradiated mouse bone marrow reconstituted with Ship-/- hematopoietic cells shows reduction in the immature and mature forms of B cells. ${ }^{32}$ Moreover, Ship-/- cells were more viable and had better survival owing to the activation of MAPK and AKT pathways. ${ }^{31}$ CCAAT enhancer-binding protein beta $(\mathrm{C} / \mathrm{EBP} \beta)$ is a mediator of the IL-6 signaling pathway; IL-6 activates C/EBP $\beta$ through the phosphorylation of MAPK..$^{33}$ In addition, C/EBP $\beta$ expression varies during differentiation of myeloid and plasma cells, ${ }^{34}$ suggesting its involvement in myeloid and lymphoid maturation. Costinean et al. showed that SHIP and $\mathrm{C} / \mathrm{EBP} \beta$ protein expression is diminished in the miR-155 transgenic pre-B lymphocytes. ${ }^{30}$ SHIP is gradually down-regulated in the preleukemic and leukemic pre-B cells, whereas C/EBP $\beta$, even though it maintains the same level of expression in preleukemic mice, is markedly down-regulated in leukemic pre-B cells. In conclusion, miR-155 transgene shows maximum expression in the pre-B stage, in which it down-regulates both SHIP and C/EBP $\beta$, two inhibitors of IL-6. This blocks B cell differentiation and might also induce a reactive proliferation of the myeloid line. The B cell precursors, arrested in their development, proliferate and are the origin of malignant leukemias with a mixed immunophenotype $\mathrm{B} 220^{\mathrm{low}} / \mathrm{IgM}^{-}$and $\mathrm{B} 220^{\mathrm{low}} / \mathrm{IgM}^{+}$. Independ ently, 0'Connell et al. ${ }^{23}$ showed that in their bone marrow adoptive transfer model, the myeloproliferative phenotype can be almost completely reproduced by knocking down SHIP expression, providing further in vivo evidence of target specificity. ${ }^{23,35}$ Thus, two distinct mouse models confirmed that SHIP mRNA is an important target of miR-155 and its repression is a critical aspect of miR-155 biology.

The BIC locus was first identified as a common retroviral integration site in avian leukosis virus (ALV)-induced B cell chicken lymphomas ${ }^{36}$ which implicated BIC as a proto-oncogene. The human $B I C$ gene was subsequently isolated and characterized as a transcript that lacks an open reading frame. ${ }^{37}$ This was the first report to suggest that oncogenesis might 
derive from the miss-expression of a non-coding RNA. Based on the presence on BIC transcripts of the hairpin typical of an miRNA, miR-155 was predicted in silico and later shown to be produced from the BIC transcripts. Indeed, miR-155 is found only in cells expressing BIC transcripts. The hairpin from which miR-155 is processed represents the only evolutionary conserved sequence of the BIC gene, suggesting that BIC oncogenic functions are through miR-155. ${ }^{28,38}$ To gain insights into miR155 physiological functions, transgenic as well as BIC/miR-155 knockout mice were developed. They showed that miR-155 is involved in almost all steps of B cell maturation and consequently modulates B cell functions. Using mice with an enhanced green fluorescent protein (EGFP) reporter gene inserted into the $B I C$ gene, it was shown that many, if not all, germinal center B cells express BIC/miR-155 at the start of the response or recurrently during proliferation and selection by antigens. As germinal centers represent sites of antibody affinity maturation and memory B cell generation in a $\mathrm{T}$ cell-dependent antibody response, the depletion of miR-155, as expected, impaired all the above. ${ }^{39,40}$ Accordingly, miR-155 ablation resulted in reduced extra follicular and germinal center responses and impaired the memory response, suggesting that miR-155 may regulate the generation of memory $\mathrm{B}$ cells..$^{38-40}$

\section{MicroRNA-17-92 cluster}

The MiR-17-92 cluster is located at human chromosome $13 q 31$, in a genomic region that is frequently amplified in lymphomas and other cancers, and the mature miRNAs encoded by this locus are highly expressed in cancer cells. ${ }^{41}$ Retroviral expression of this miRNA cluster in the hematopoietic system accelerated the onset of cMyc-mediated lymphomagenesis in a transgenic mouse model involving bone marrow reconstitution. ${ }^{42}$ To elucidate the mechanism by which the miR-17-92 cluster promotes lymphomagenesis, Xiao et al..$^{43}$ generated mice with elevated miR-17-92 expression in lymphocytes. They showed that Pten and Bim mRNAs, both of which have tumor suppressive activities, are direct targets of miRNAs in the miR-17-92 cluster, and that Pten and Bim protein expression is down-regulated upon constitutive and moderate overexpression of miR-17-92 miRNAs. In mouse B and T cells, miR-17-92 over-expression leads to a LPD, characterized by lymphoid hyperplasia in the spleen, activated lymphocytes, and lymphoid infiltration of peripheral tissues accompanied by an autoimmune phenotype, ${ }^{35,43}$ but not malignancy. A mechanism involving Bim is consistent with the findings that Bim+/- mice develop a phenotype similar to that of mice over-expressing miR-17-92 and that heterozygous deficiency of Bim, similar to over-expression of miR-17-92, cooperates with E $\mu-M y c$ in lymphomagenesis..$^{35,42}$

The miR-17-92 cluster produces a single polycistronic primary transcript that yields six individual mature miRNAs. The distinct mature miRNA sequence of these miR-17-92 components dictates the specificity of their target regulation, and ultimately determines the functional specificity. Olive et al ${ }^{44}$ reported the functional dissection of miR-17-92 in the context of B cell transformation in vivo, and revealed the essential role of miR-19 in mediating the oncogenic activity of miR-17-92. In the Eu-Myc model of Burkitt's lymphoma, miR19 is both necessary and sufficient for miR-1792 to promote c-Myc-induced B lymphomagenesis. The proliferative activity of miR-19 is mediated, at least in part, by the PI3K-AktmTOR pathway, as enforced miR-19 expression dampens the expression of Pten, thus activating Akt-mTOR signaling to promote cell survival. ${ }^{44}$ Independently, similar findings were also described by Ventura's laboratory with a different model based on a conditional loss of function of miR-17-92. ${ }^{45}$

\section{Concluding remarks and future developments}

This review focused on the currently available mouse models of miRNAs in cancer of immunological origin but this is a field of investigation in rapid expansion and it is likely that many more models will be published in the near future. The discovery of miRNA dysregulation in leukemia and other malignancies offered to cancer researchers an unprecedented level of precision to study the complexities and intricacies of cancer pathways. ${ }^{46}$ The creation of ever more accurate mouse models of human hematopoietic cancers based on miRNA dysregulation is likely to be of benefit in a special way given the particular susceptibility of mice to leukemia in comparison to solid malignancies. Indeed, a future development will be the production of transgenic, knockout, and knockin in vivo models for all those miRNAs previously identified as involved in leukemias, to establish their contribution to malignant transformation in immune cells. An additional development will be characterized by the crosses of these new miRNA models with engineered mice of oncogenes and tumor suppressors targeted by these miRNAs to recreate in vivo the complex, but more authentic relationship between protein-coding genes and non protein-coding genes belonging to the same cancer pathway. Interestingly, many existing models of oncogenes and tumor suppressors will be reconsidered in the light of new knowledge of miRNA signal pathways. Finally, the therapeutic use of miRNAs and anti-miRNAs in new and established mouse

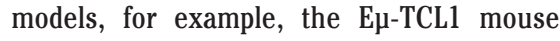
model of aggressive CLL, ${ }^{47-49}$ will provide the necessary insights for a shift from conventional approaches to more targeted therapies in human leukemia and other cancers.

\section{References}

1. Bartel DP. MicroRNAs: genomics, biogenesis, mechanism, and function. Cell 2004; 116:281-97.

2. Zhang $\mathrm{H}$, Chen $\mathrm{Y}$. New insight into the role of miRNAs in leukemia. Sci China C Life Sci 2009;52:224-31.

3. Fabbri M, Ivan M, Cimmino A, et al. Regulatory mechanisms of microRNAs involvement in cancer. Expert Opin Biol Ther 2007;7:1009-19.

4. Calin GA, Dumitru CD, Shimizu M, et al. Frequent deletions and down-regulation of micro-RNA genes miR15 and miR16 at $13 q 14$ in chronic lymphocytic leukemia. Proc Natl Acad Sci USA 2002;99:15524-9.

5. Calin GA, Liu CG, Sevignani C, et al. MicroRNA profiling reveals distinct signatures in B cell chronic lymphocytic leukemias. Proc Natl Acad Sci USA 2004; 101:11755-60.

6. Volinia S, Calin GA, Liu CG, et al. A micro RNA expression signature of human solid tumors defines cancer gene targets. Proc Natl Acad Sci USA 2006;103:2257-61.

7. Pichiorri F, Suh SS, Ladetto M, et al. MicroRNAs regulate critical genes associated with multiple myeloma pathogenesis. Proc Natl Acad Sci USA 2008;105:12885-90.

8. Holland EC. Preface. In: Mouse models of human cancers. Holland EC, ed. Noboken, NJ: Wiley-Liss; 2004, p vii.

9. Fabbri M, Croce CM, Calin GA. MicroRNAs in the ontogeny of leukemias and lymphomas. Leuk Lymphoma 2009;50:160-70.

10. Cimmino A, Calin GA, Fabbri M, et al. miR15 and miR-16 induce apoptosis by targeting BCL2. Proc Natl Acad Sci USA 2005; 102:13944-9.

11. Scaglione BJ, Salerno E, Balan M, et al. Murine models of chronic lymphocytic leukaemia: role of microRNA-16 in the New Zealand Black mouse model. Br J Haematol 2007;139:645-57.

12. Pekarsky Y, Zanesi N, Aqeilan RI, et al. Animal models for chronic lymphocytic leukemia. J Cell Biochem 2007;100:110918.

13. Raveche ES. Possible immunoregulatory role for CD5+ B cells. Clin Immunol Immunopathol 1990;56:135-50.

14. Liu Y, Corcoran M, Rasool 0, et al. Cloning 
of two candidate tumor suppressor genes within a $10 \mathrm{~kb}$ region on chromosome $13 q 14$, frequently deleted in chronic lymphocytic leukemia. Oncogene 1997;15: 2463-73.

15. Bullrich F, Fujii H, Calin G, et al. Characterization of the 13q14 tumor suppressor locus in CLL: identification of ALT1, an alternative splice variant of the LEU2 gene. Cancer Res 2001;61:6640-8.

16. Corcoran MM, Rasool O, Liu Y, et al. Detailed molecular delineation of $13 q 14.3$ loss in B-cell chronic lymphocytic leukemia. Blood 1998;91:1382-90.

17. Calin GA, Ferracin M, Cimmino A, et al. A MicroRNA signature associated with prognosis and progression in chronic lymphocytic leukemia. N Engl J Med 2005;353: 1793-801.

18. Raveche ES, Salerno E, Scaglione BJ, et al. Abnormal microRNA-16 locus with synteny to human 13q14 linked to CLL in NZB mice. Blood 2007;109:5079-86.

19. Klein U, Lia M, Crespo $M$, et al. The DLEU2/miR-15a/16-1 cluster controls B cell proliferation and its deletion leads to chronic lymphocytic leukemia. Cancer Cell 2010;17:28-40.

20. Migliazza A, Bosch F, Komatsu H, et al. Nucleotide sequence, transcription map, and mutation analysis of the $13 q 14$ chromosomal region deleted in B-cell chronic lymphocytic leukemia. Blood 2001;97: 2098-104.

21. Salerno E, Scaglione BJ, Coffman FD, et al. Correcting miR-15a/16 genetic defect in New Zealand Black mouse model of CLL enhances drug sensitivity. Mol Cancer Ther 2009;8:2684-92.

22. Peng B, Sherr DH, Mahboudi F, et al. A cultured malignant B-1 line serves as a model for Richter's syndrome. J Immunol 1994; 153:1869-80.

23. O'Connell RM, Chaudhuri AA, Rao DS, et al. Inositol phosphatase SHIP1 is a primary target of miR-155. Proc Natl Acad Sci USA 2009;106:7113-8.

24. Kluiver J, Poppema S, de Jong D, et al. BIC and miR-155 are highly expressed in Hodgkin, primary mediastinal and diffuse large B cell lymphomas. J Pathol 2005; 207:243-9.

25. Calin GA, Croce CM. Genomics of chronic lymphocytic leukemia microRNAs as new players with clinical significance. Semin
Oncol 2006;33:167-73.

26. O'Connell RM, Rao DS, Chaudhuri AA, et al. Sustained expression of microRNA-155 in hematopoietic stem cells causes a myeloproliferative disorder. J Exp Med 2008;205:585-94.

27. Garzon R, Garofalo M, Martelli MP, et al. Distinctive microRNA signature of acute myeloid leukemia bearing cytoplasmic mutated nucleophosmin. Proc Natl Acad Sci USA 2008;105:3945-50.

28. Eis PS, Tam W, Sun L, et al. Accumulation of miR-155 and BIC RNA in human B cell lymphomas. Proc Natl Acad Sci USA 2005; 102:3627-32.

29. Costinean S, Zanesi N, Pekarsky Y, et al. Pre-B cell proliferation and lymphoblastic leukemia/high-grade lymphoma in E(mu)miR155 transgenic mice. Proc Natl Acad Sci USA 2006;103:7024-9.

30. Costinean S, Sandhu SK, Pedersen IM, et al. Src homology 2 domain-containing inositol-5-phosphatase and CCAAT enhancer-binding protein beta are targeted by miR-155 in B cells of Emicro-MiR-155 transgenic mice. Blood 2009;114:1374-82.

31. Helgason CD, Kalberer CP, Damen JE, et al. A dual role for Src homology 2 domaincontaining inositol-5-phosphatase (SHIP) in immunity: aberrant development and enhanced function of $B$ lymphocytes in ship -/- mice. J Exp Med 2000;191:781-94.

32. Liu Q, Oliveira-Dos-Santos AJ, Mariathasan S, et al. The inositol polyphosphate 5 -phosphatase ship is a crucial negative regulator of B cell antigen receptor signaling. J Exp Med 1998;188:1333-42.

33. Trautwein C, Caelles C, van der Geer P, et al. Transactivation by NF-IL6/LAP is enhanced by phosphorylation of its activation domain. Nature 1993;364:544-7.

34. Natsuka S, Akira S, Nishio Y, et al. Macrophage differentiation-specific expression of NF-IL6, a transcription factor for interleukin-6. Blood 1992;79:460-6.

35. O'Connell RM, Rao DS, Chaudhuri AA, Baltimore D. Physiological and pathological roles for microRNAs in the immune system. Nat Rev Immunol;10:111-22.

36. Clurman BE, Hayward WS. Multiple protooncogene activations in avian leukosis virus-induced lymphomas: evidence for stage-specific events. Mol Cell Biol 1989; 9:2657-64.

37. Tam W, Ben-Yehuda D, Hayward WS. bic, a novel gene activated by proviral insertions in avian leukosis virus-induced lymphomas, is likely to function through its noncoding RNA. Mol Cell Biol 1997;17: 1490-502.

38. Tili E, Croce CM, Michaille JJ. miR-155: on the crosstalk between inflammation and cancer. Int Rev Immunol 2009;28:264-84.

39. Thai TH, Calado DP, Casola S, et al. Regulation of the germinal center response by microRNA-155. Science 2007; 316:604-8.

40. Rodriguez A, Vigorito E, Clare S, et al. Requirement of bic/microRNA-155 for normal immune function. Science 2007;316: 608-11.

41. Lu J, Getz G, Miska EA, et al. MicroRNA expression profiles classify human cancers. Nature 2005;435:834-8.

42. He L, Thomson JM, Hemann MT, et al. A microRNA polycistron as a potential human oncogene. Nature 2005;435:828-33.

43. Xiao C, Srinivasan L, Calado DP, et al. Lymphoproliferative disease and autoimmunity in mice with increased miR-17-92 expression in lymphocytes. Nat Immunol 2008;9:405-14.

44. Olive V, Bennett MJ, Walker JC, et al. miR19 is a key oncogenic component of mir17-92. Genes Dev 2009;23:2839-49.

45. Mu P, Han YC, Betel D, et al. Genetic dissection of the miR-17 92 cluster of microRNAs in Myc-induced B-cell lymphomas. Genes Dev 2009;23:2806-11.

46. Croce CM. Causes and consequences of microRNA dysregulation in cancer. Nat Rev Genet 2009;10:704-14.

47. Bichi R, Shinton SA, Martin ES, et al. Human chronic lymphocytic leukemia modeled in mouse by targeted TCL1 expression. Proc Natl Acad Sci USA 2002; 99:6955-60.

48. Zanesi N, Aqeilan R, Drusco A, et al. Effect of Rapamycin on Mouse Chronic Lymphocytic Leukemia and the Development of Nonhematopoietic Malignancies in EmuTCL1 Transgenic Mice. Cancer Res 2006;66:915-20.

49. Efanov A, Zanesi N, Nazaryan N, et al. CD5+CD23+ leukemic cell populations in TCL1 transgenic mice show significantly increased proliferation and Akt phosphorylation. Leukemia 2010;24:970-5. 